\title{
Estado nutricional e sua associação com risco cardiovascular e síndrome metabólica em idosos ${ }^{1}$
}

\author{
Nutritional status and its association with \\ cardiovascular risk and metabolic \\ syndrome in the elderly
}

Fernanda SCHERER²

José Luiz da Costa VIEIRA3

\section{RE S U M O}

\section{Objetivo}

Caracterizar o estado nutricional de uma população de idosos e verificar sua associação com fatores de risco cardiovascular e síndrome metabólica.

\section{Métodos}

Foram avaliados o estado nutricional e prevalências de fatores de risco cardiovascular de síndrome metabólica em amostra de idosos em município da região central do Rio Grande do Sul. O estado nutricional foi determinado por meio de índice de massa corporal, conforme classificação de Lipschitz/1994. O diagnóstico de síndrome metabólica foi obtido por meio dos critérios da International Diabetes Federation.

\section{Resultados}

Entre os idosos avaliados, a prevalência de excesso de peso foi de $42 \%$ entre os homens e de $50 \%$ entre as mulheres. As prevalências de hipertensão arterial sistêmica, diabetes mellitus, síndrome metabólica, obesidade central, lipoproteína de alta densidade - colesterol baixo e triglicerídeos elevados foram maiores naqueles com excesso de peso de ambos os sexos. Usando como referência os com estado nutricional normal, os portadores de excesso de peso apresentaram aumento no risco de hipertensão arterial sistêmica, diabetes mellitus e síndrome metabólica, com razão de chances de, respectivamente, 3,3 (IC95\%: 1,7 a 6,4), 3,3 (IC95\%: 1,48 a 7,11$)$ e 6,9 (IC95\%: 3,2 a 14,6), enquanto aqueles com magreza tiveram tendência à razão de chances reduzidas para essas doenças.

1 Artigo elaborado a partir da dissertação de F. SCHERER, intitulada "Estado nutricional e sua associação com fatores de risco cardiovascular e síndrome metabólica em idosos". Pontifícia Universidade Católica do Rio Grande do Sul; 2008.

2 Pontifícia Universidade Católica do Rio Grande do Sul, Instituto de Geriatria e Gerontologia. Av. Ipiranga, 6681, Partenon, 90619-900, Porto Alegre, RS, Brasil. Correspondência para/Correspondence to: F. SCHERER. E-mail:<frscherer@ibest.com.br>.

3 Fundação Universitária de Cardiologia, Instituto de Cardiologia, Curso de Pós-Graduação em Ciências da Saúde. Porto Alegre, RS, Brasil. 
348 | F. SCHERER \& J.L.C. VIEIRA

\section{Conclusão}

As razões de chances de apresentar hipertensão arterial sistêmica, diabetes mellitus e síndrome metabólica na população de idosos da pesquisa estão associadas ao estado nutricional definido pelo índice de massa corporal, sendo progressivamente maiores naqueles com excesso de peso e menores nos com magreza, independente de gênero e idade.

Termos de indexação: Estado nutricional. Fatores de risco cardiovascular. Idoso. Índice de massa corporal. Síndrome metabólica.

\section{A B S T R A C T}

\section{Objective}

This study aimed to characterize the nutritional status of an elderly population and verify its relationship with cardiovascular risk factors and metabolic syndrome.

\section{Methods}

Nutritional status and prevalence of cardiovascular risk factors and metabolic syndrome were assessed in an elderly population from a city in the state of Rio Grande do Sul, in the South of Brazil. Body mass index, according to the Lipschitz classification of 1994, was used to determine their nutritional status. Metabolic syndrome diagnosis was based on the International Diabetes Federation criteria.

\section{Results}

Among the studied elderly, $42 \%$ of the males and 50\% of the females were overweight. Hypertension, diabetes mellitus, metabolic syndrome, central obesity, low high density lipoprotein-cholesterol levels and high triglyceride levels were more common in overweight individuals than in normal weight individuals of both genders. Using the normal weight individuals as reference, the overweight were at greater risk of systemic hypertension, diabetes mellitus and metabolic syndrome, with an odds ratio of 3.3 (CI95\%: 1.7 to 6.4), 3.3 (C195\%: 1.48 to 7.1) and 6.9 (C195\%: 3.2 to 14.6) respectively, while the underweight tended towards a lower odds ratio for the same diseases.

\section{Conclusion}

The odds ratios for the presence of hypertension, diabetes mellitus and metabolic syndrome were associated with nutritional status determined by body mass index. The odds ratios are progressively higher in the overweight and lower in the underweight, regardless of age and gender.

Indexing terms: Nutritional status. Cardiovascular risk factors. Elderly. Body mass índex. Metabolic syndrome.

\section{N T R O D U ÇÃ O}

A obesidade desencadeia e/ou exacerba Doenças Crônico-Degenerativas (DCD), como as Doenças Cardiovasculares (DCV), Hipertensão Arterial Sistêmica (HAS), Diabetes Mellitus (DM), dislipidemias - hipertrigliceridemia e níveis de Lipoproteína de Alta Densidade-Colesterol (HDL-c) diminuídos - e Síndrome Metabólica (SM). A obesidade do tipo central pode ser responsabilizada por aproximadamente $20 \%$ dos casos diagnosticados de infarto agudo do miocárdio, independentemente de outros fatores de risco ${ }^{1}$. A Associação Norte-Americana de Cardiologia tem classificado a obesidade como principal fator de risco modificável para DCV e para SM². Sabe-se que a DM está intimamente associada ao aumento de risco cardiovascular. A SM, caracterizada por obesidade central, dislipidemia, hiperglicemia e hipertensão, é hoje um dos maiores desafios para a saúde pública em todo o mundo, por associar-se a importante risco para DCV e DM ${ }^{3}$. Importante destacar a associação da SM com a DCV, aumentando a mortalidade cardiovascular em cerca de 2,5 vezes e a mortalidade total em cerca de 1,5 vezes ${ }^{4}$.

Considerando que a obesidade e a SM aumentam sua prevalência com o avanço da idade e que os idosos são do grupo da população com maior prevalência de eventos cardiovasculares, a 
importância do tema se torna ainda maior, já que a população idosa vem crescendo em todo o mundo, principalmente em países em desenvolvimento como o Brasil 5 .

Embora a prevalência de obesidade venha aumentando, existem controvérsias acerca de seu diagnóstico e tratamento em idosos ${ }^{6}$. Dados relativos à prevalência de obesidade e outros fatores de risco cardiovasculares na população geriátrica em nosso meio são escassos. Identificar a associação de estado nutricional e fatores de risco cardiovascular nessa população adquire grande importância para medidas de controle de risco, pois pode ajudar a identificar aqueles sob maior risco dentre um grupo já com risco elevado. Dentro dessa visão, este estudo tem por objetivo avaliar o estado nutricional de idosos de uma população do interior do Estado do Rio Grande do Sul (RS) e verificar sua associação com fatores de risco cardiovascular e SM.

\section{M É T O D O S}

Foi realizado estudo transversal, de base populacional, em Marques de Souza, município de colonização alemã e italiana, localizado na região central do Estado do Rio Grande do Sul, e que tem como principais atividades a agricultura e a pecuária, sendo, por essas características, semelhante à grande parcela do interior desse estado. A população total do município, pelo censo de 2000, era de 4241 habitantes, dos quais 777 eram idosos, sendo, segundo dados não publicados da Secretaria Municipal de Saúde, 424 mulheres e 353 homens, morando 363 na área rural e 414 na urbana. Foram candidatos a participarem do estudo todos idosos habitantes do município cadastrados pelos agentes de saúde.

O tamanho amostral foi calculado em 195 idosos para avaliar a prevalência de obesidade dessa população com margem de erro de 0,05, tendo por base prevalência de obesidade nesse grupo etário de $19 \%{ }^{7}$. Foram então convidados a participar do estudo $25 \%$ da população de idosos, selecionados aleatoriamente após estratificação por gênero, faixa etária (60 a 69 anos, 70 a 79 anos e 80 anos ou mais) e local de moradia (rural e urbano). Os dados foram coletados em março de 2007. O projeto foi aprovado pelo Comitê de Ética Científica do Instituto de Geriatria e Gerontologia e pelo Comitê de Ética em Pesquisa da Pontifícia Universidade Católica do Rio Grande do Sul, registro CEP 06/03559.

Após assinarem o termo de consentimento livre e esclarecido, os idosos que aceitaram participar do estudo responderam a um questionário estruturado, no qual foram registrados dados sociodemográficos e presença de fatores de risco cardiovascular. Medidas clínicas - peso, estatura, circunferência abdominal e pressão arterial - foram realizadas por pesquisadores treinados. $\mathrm{O}$ peso e a estatura foram avaliados em balança antropométrica com estadiômetro acoplado Welmy ${ }^{\circledR}$. O cálculo do Índice de Massa Corporal (IMC) foi efetuado considerando o peso em quilos dividido pelo quadrado da altura em metros e classificado conforme critério de Lipschitz ${ }^{8}$. A circunferência abdominal foi medida com fita métrica inelástica, no ponto médio entre as últimas costelas e a crista ilíaca ântero-superior, no final do movimento respiratório de expiração, em pé. A pressão arterial foi verificada nos dois braços, com o paciente sentado, após 10 minutos de descanso, com um esfigmomanômetro aneroide previamente calibrado, sendo utilizada a média dos dois valores encontrados.

As coletas de sangue foram realizadas com jejum de 12 horas e posteriormente analisadas no Laboratório Hermann Ltda de Lajeado, com certificação do Conselho Regional de Farmácia do RS. Para dosagem sérica de glicemia, colesterol total, HDL-C e triglicerídeos, foram usados os materiais da marca Labtest ${ }^{\circledR}$. As amostras foram analisadas por método enzimático colorimétrico em equipamento de semiautomação.

O critério utilizado para o diagnóstico de SM foi o do International Diabetes Federation (IDF), sendo necessária a presença da circunferência abdominal alterada como fator pré-requisito para o diagnóstico da síndrome e a presença adicional 
de dois ou mais dos seguintes componentes: glicose plasmática em jejum $\geq 100 \mathrm{mg} / \mathrm{dL}$ ou diagnóstico prévio de diabetes mellitus tipo 2; pressão sistólica $\geq 130 \mathrm{mmHg}$ ou diastólica $\geq 85 \mathrm{mmHg}$ ou uso de anti-hipertensivo; HDL-C $<40 \mathrm{mg} / \mathrm{dL}$ nos homens e $<50 \mathrm{mg} / \mathrm{dL}$ nas mulheres ou uso de medicação específica para aumentar HDL-C; triglicerídeos $\geq 150 \mathrm{mg} / \mathrm{dL}$ ou uso de medicação específica para tratar hipertrigliceridemia. Como a população era na sua totalidade de descendentes de europeus, foram utilizados pontos de corte de $94 \mathrm{~cm}$ para os homens e $80 \mathrm{~cm}$ para as mulheres ${ }^{3}$.

Os dados quantitativos foram descritos por média e desvio-padrão e os categóricos, por contagens e percentuais. Para a comparação entre os gêneros, foram utilizado o teste $t$ de Student e o teste do qui-quadrado. Para a comparação entre as categorias de estado nutricional, utilizaram-se a ANOVA e o teste do qui-quadrado. ANCOVA com médias ajustadas para idade foi utilizada para avaliar a associação de fatores de risco para SM com a faixa do estado nutricional definida pelo IMC nos dois gêneros. Para calcular a razão de chances (odds ratio) do diagnóstico de HAS, DM e SM em cada uma das categorias de estado nutricional, foi utilizada regressão logística, em modelo ajustado para idade e gênero. O nível de significância utilizado foi definido em $\alpha=0,05$ bi-caudal. Para a análise estatística, utilizou-se o programa estatístico SPSS, versão13 (SPSS Chicago, IL).

\section{RES U LTA D OS}

Participaram do estudo 199 idosos, 102 mulheres e 97 homens, com idade média dos homens de 71,4 anos e das mulheres de 69,9 anos (Tabela 1). Em relação ao nível de escolaridade, $87 \%$ dos homens e $88 \%$ das mulheres apresentavam ensino fundamental incompleto. A faixa de renda com maior prevalência foi entre um e três salários-mínimos per capita, tanto para homens (44\%) como para mulheres (57\%). Moravam na zona urbana $54 \%$ dos homens e $69 \%$ das mulheres.

Em relação ao estado nutricional, o excesso de peso foi constatado em $42 \%$ dos homens e $50 \%$ das mulheres, enquanto $22 \%$ dos homens e $13 \%$ das mulheres apresentavam magreza. $A$ prevalência de DM não foi diferente entre os dois gêneros (22\% nos homens vs. $23 \%$ nas mulheres, $p=0,87$ ), mas a SM foi menos prevalente entre os homens do que entre as mulheres ( $26 \%$ vs. $44 \%$, respectivamente, $p=0,008)$. Considerando os limites estabelecidos para cada gênero, a prevalência de obesidade central e a de níveis de HDL-C baixos foram maiores no gênero feminino (Tabela 2).

Tabela 1. Características demográficas dos participantes homens e mulheres. Marques de Souza (RS), 2007.

\begin{tabular}{|c|c|c|c|c|c|}
\hline Característica & \multicolumn{2}{|c|}{ Homens $n=97$} & \multicolumn{2}{|c|}{ Mulheres $n=102$} & $p$ \\
\hline Idade (anos) & \multicolumn{2}{|c|}{$M=69,9, D P=6,7$} & \multicolumn{2}{|c|}{$M=71,4, D P=8,1$} & 0,15 \\
\hline Escolaridade & $n$ & $\%$ & $n$ & $\%$ & \\
\hline Analfabeto & 6 & 6 & 6 & 6 & \\
\hline Fundamental incompleto & 84 & 87 & 90 & 88 & \\
\hline Fundamental completo ou mais & 7 & 7 & 6 & 6 & 0,95 \\
\hline Renda mensal per capita & $n$ & $\%$ & $n$ & $\%$ & \\
\hline Até 1 salário-mínimo & 36 & 37 & 45 & 44 & \\
\hline$>1$ e $<3$ salários-mínimos & 55 & 57 & 45 & 44 & \\
\hline >3 salários-mínimos & 6 & 6 & 12 & 12 & 0,15 \\
\hline Local de moradia & $n$ & $\%$ & $n$ & $\%$ & \\
\hline Área rural & 45 & 46 & 32 & 31 & \\
\hline Área urbana & 52 & 54 & 70 & 69 & $0,04^{*}$ \\
\hline
\end{tabular}

Os dados são apresentados como M: média, DP: desvio-padrão ou número (\%); ${ }^{*} p<0,05$. 
Tabela 2. Características clínicas e laboratoriais dos participantes homens e mulheres. Marques de Souza (RS), 2007.

\begin{tabular}{|c|c|c|c|c|c|}
\hline \multirow[t]{2}{*}{ Característica } & \multicolumn{2}{|c|}{ Homens $n=97$} & \multicolumn{2}{|c|}{ Mulheres $n=102$} & \multirow[t]{2}{*}{$p$} \\
\hline & $M$ & $D P$ & $M$ & $D P$ & \\
\hline Peso (kg) & 74,9 & 14,8 & 65,3 & 10,7 & $<0,001^{*}$ \\
\hline IMC (kg/m²) & 26,3 & 4,9 & 26,9 & 4,4 & 0,36 \\
\hline Estado nutricional & $n$ & $\%$ & $n$ & $\%$ & \\
\hline Magreza & 21 & 22 & 13 & 13 & \\
\hline Normal & 35 & 36 & 38 & 37 & \\
\hline Excesso de peso & 41 & 42 & 51 & 50 & 0,126 \\
\hline \multirow[t]{2}{*}{ Circunferência da cintura $(\mathrm{cm})$} & $M$ & $D P$ & $M$ & $D P$ & \\
\hline & 93,5 & 12,8 & 86,8 & 11,3 & $<0,001^{*}$ \\
\hline \multirow[t]{2}{*}{ Tabagismo } & $n$ & $\%$ & $n$ & $\%$ & \\
\hline & 22 & 23 & 0 & 0 & $<0,001^{*}$ \\
\hline HAS & 46 & 47 & 60 & 59 & 0,12 \\
\hline DM & 21 & 22 & 24 & 23 & 0,87 \\
\hline SM & 25 & 26 & 45 & 44 & $0,008^{*}$ \\
\hline \multirow[t]{2}{*}{ Glicemia (mg/dL) } & $M$ & $D P$ & $M$ & $D P$ & \\
\hline & 91 & 20 & 93 & 30 & 0,55 \\
\hline Perfil lipídico (mg/dL) & $M$ & $D P$ & $M$ & $D P$ & \\
\hline Colesterol total & 207 & 47 & 234 & 56 & $<0,001^{*}$ \\
\hline LDL colesterol & 132 & 43 & 150 & 47 & $0,006^{*}$ \\
\hline HDL colesterol & 52 & 15 & 56 & 17 & 0,08 \\
\hline \multirow[t]{2}{*}{ Triglicerídeos } & 118 & 64 & 135 & 69 & 0,07 \\
\hline & $n$ & $\%$ & $n$ & $\%$ & \\
\hline Obesidade central $^{* *}$ & 44 & 45 & 73 & 72 & $<0,001^{*}$ \\
\hline PA elevada ${ }^{\star \star \star}$ & 46 & 47 & 60 & 59 & 0,12 \\
\hline Glicemia elevada ${ }^{* \star \star \star *}$ & 21 & 22 & 24 & 24 & 0,87 \\
\hline 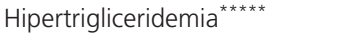 & 20 & 21 & 30 & 29 & 0,19 \\
\hline HDL-C baixo***** & 15 & 16 & 37 & 36 & $0,001^{*}$ \\
\hline
\end{tabular}

Os dados são apresentados como M:média; DP: desvio-padrão; n: número; \%: porcentagem.

*Diferença significativa; ${ }^{* *}$ Obesidade central: circunferência da cintura $>80 \mathrm{~cm}$ em mulheres e $>94 \mathrm{~cm}$ em homens; ${ }^{* * *}$ Pressão arterial elevada: sistólica $\geq 130 \mathrm{mmHg}$, diastólica $\geq 85 \mathrm{mmHg}$, uso de medicação anti-hipertensiva; ${ }^{* * * *}$ Glicemia de jejum elevada: $\geq 100 \mathrm{mg} / \mathrm{dL}$ ou diagnóstico médico de diabetes tipo $2 ;{ }^{* * * * *}$ Hipertrigliceridemia: $>150 \mathrm{mg} / \mathrm{dL}$ em jejum; ${ }^{* * * * * *} \mathrm{HDL}-\mathrm{C}$ baixo: $<40 \mathrm{mg} / \mathrm{dL}$ em homens e $<50 \mathrm{mg} / \mathrm{dL}$ em mulheres, em jejum.

IMC: índice de massa corporal; PA: pressão arterial; HDL-C: colesterol da lipoproteína de alta densidade.

As características demográficas de faixa etária, renda per capita e local de moradia, estratificadas por gênero, não demonstraram nenhuma associação com as categorias de estado nutricional.

Em relação ao perfil lipídico, observa-se que os homens com excesso de peso apresentaram níveis significativamente maiores de LDL-C e triglicerídeos e menores de HDL-C em relação àqueles com estado nutricional classificado como magreza e normal, diferença não encontrada entre as mulheres (Tabela 3).
As prevalências de HAS, SM, DM e de todos os componentes da síndrome metabólica tiveram, em ambos os gêneros, relação significativamente maior com excesso de peso quando comparados com os idosos classificados como magros e normais.

Quando comparados os idosos, de ambos os gêneros, de estado nutricional normal aos com excesso de peso, a razão de chances de estes últimos apresentarem HAS foi de 3,3 (IC 95\%\%: 1,7 a 6,4), DM, 3,3 (IC 95\%\%: 1,48 a 7,11) e SM, 6,9 (IC 95\% \%: 3,2 a 14,6). Quando compa- 
ramos o risco de os idosos magros apresentarem as mesmas doenças, encontramos uma tendência à redução da razão de chance para HAS e DM respectivamente de 0,42 (IC95\%\%: 0,17 a 1,06) e 0,37 (IC 95\% \%: 0,08 a 1,78). Não foi encontrado nenhum caso de SM entre os idosos classificados como magros (Tabela 4).
DISCUSS Ã O

Nesta amostra representativa de parcela da população de idosos do interior do Rio Grande do Sul, com a determinação do estado nutricional por meio do IMC, utilizando-se os pontos de corte normalmente utilizados para essa população, as

Tabela 3. Características clínicas dos participantes homens e mulheres de acordo com o estado nutricional. Marques de Souza (RS), 2007.

\begin{tabular}{|c|c|c|c|c|c|c|c|c|c|c|c|c|c|c|}
\hline \multirow{3}{*}{ Característica } & \multicolumn{6}{|c|}{ Homens } & \multirow{3}{*}{$p$} & \multicolumn{6}{|c|}{ Mulheres } & \multirow{3}{*}{$p$} \\
\hline & \multicolumn{2}{|c|}{$\begin{array}{c}\text { Magreza } \\
n=21\end{array}$} & \multicolumn{2}{|c|}{$\begin{array}{l}\text { Normal } \\
n=35\end{array}$} & \multicolumn{2}{|c|}{$\begin{array}{l}\text { Excesso de } \\
\text { peso } n=41\end{array}$} & & \multicolumn{2}{|c|}{$\begin{array}{l}\text { Magreza } \\
n=13\end{array}$} & \multicolumn{2}{|c|}{$\begin{array}{c}\text { Normal } \\
n=38\end{array}$} & \multicolumn{2}{|c|}{$\begin{array}{l}\text { Excesso de } \\
\text { peso } n=51\end{array}$} & \\
\hline & $\mathrm{M}$ & $\mathrm{DP}$ & $\mathrm{M}$ & DP & $\mathrm{M}$ & DP & & $\mathrm{M}$ & $\mathrm{DP}$ & $\mathrm{M}$ & DP & $\mathrm{M}$ & DP & \\
\hline Glicemia (mg/dL) & 85 & 11 & 92 & 25 & 92 & 19 & 0,39 & 84 & 10 & 86 & 11 & 100 & 40 & $0,04^{*}$ \\
\hline Perfil Lipídico (mg/dL) & M & $D P$ & M & $D P$ & M & $D P$ & & M & $D P$ & $\mathrm{M}$ & $D P$ & M & $D P$ & \\
\hline Colesterol total & 198 & 44 & 199 & 43 & 220 & 51 & 0,09 & 248 & 63 & 229 & 56 & 234 & 53 & $0,57^{*}$ \\
\hline LDL-colesterol & 118 & $42^{3}$ & 124 & 38 & 145 & $45^{1}$ & $0,02^{*}$ & 167 & 62 & 148 & 53 & 146 & 37 & 0,33 \\
\hline HDL-colesterol & 61 & $14^{3}$ & 54 & $15^{3}$ & 46 & $14^{1,2}$ & $<0,001^{*}$ & 59 & 16 & 56 & 19 & 55 & 16 & 0,77 \\
\hline \multirow[t]{2}{*}{ Triglicerídeos } & 94 & $38^{3}$ & 103 & $59^{3}$ & 142 & $71^{1,2}$ & $0,04^{*}$ & 107 & 30 & 128 & 67 & 146 & 75 & 0,14 \\
\hline & $n$ & $\%$ & $n$ & $\%$ & $\mathrm{n}$ & $\%$ & & $n$ & $\%$ & $n$ & $\%$ & $n$ & $\%$ & \\
\hline Tabagismo & 9 & 43 & 8 & 23 & 5 & 12 & $0,04^{*}$ & 0 & 0 & 0 & 0 & 0 & 0 & \\
\hline HAS & 4 & 19 & 12 & 34 & 30 & 73 & $<0,001^{*}$ & 4 & 31 & 20 & 53 & 36 & 71 & $0,04^{*}$ \\
\hline Diabetes mellitus & 1 & 5 & 7 & 20 & 13 & 32 & $0,006^{*}$ & 1 & 8 & 4 & 10 & 19 & 37 & $0,001^{*}$ \\
\hline Síndrome metabólica & 0 & 0 & 2 & 6 & 23 & 56 & $<0,001^{*}$ & 0 & 0 & 13 & 34 & 32 & 63 & $<0,001^{*}$ \\
\hline $\begin{array}{l}\text { Componentes da } \\
\text { Síndrome Metabolica }\end{array}$ & $n$ & $\%$ & $\mathrm{n}$ & $\%$ & $\mathrm{n}$ & $\%$ & & $n$ & $\%$ & $n$ & $\%$ & $\mathrm{n}$ & $\%$ & \\
\hline Obesidade central $^{\star *}$ & 0 & 0 & 10 & 29 & 34 & 83 & $<0,001^{*}$ & 1 & 8 & 23 & 61 & 49 & 96 & $<0,001^{*}$ \\
\hline PA elevada*** & 14 & 67 & 28 & 80 & 41 & 100 & $<0,001^{*}$ & 10 & 77 & 32 & 84 & 47 & 92 & $0,04^{*}$ \\
\hline Glicemia elevada ${ }^{* \star \star}$ & 1 & 5 & 7 & 20 & 14 & 34 & $0,003^{*}$ & 1 & 8 & 4 & 11 & 19 & 37 & $0,001^{*}$ \\
\hline Hipertrigliceridemia $^{* \star \star \star \star}$ & 2 & 10 & 3 & 9 & 15 & 37 & $0,002^{*}$ & 2 & 15 & 8 & 21 & 20 & 39 & $0,01^{*}$ \\
\hline HDL-C baixo ${ }^{* * * * *}$ & 0 & 0 & 4 & 11 & 11 & 27 & $0,002^{\star}$ & 3 & 23 & 14 & 37 & 20 & 39 & 0,07 \\
\hline
\end{tabular}

Os dados são apresentados como M: média; DP: desvio-padrão; n: número; \%: porcentagem.

${ }^{1} p<0,05$ para comparação com grupo magreza; ${ }^{2} p<0,05$ para comparação com grupo normal; ${ }^{3} p<0,05$ para comparação com grupo excesso de peso. " $p<0,05$; ${ }^{* *}$ Obesidade central: circunferência da cintura $>80 \mathrm{~cm}$ em mulheres e $>94 \mathrm{~cm}$ em homens; ${ }^{* * *}$ Pressão arterial elevada: sistólica $\geq 130 \mathrm{mmHg}$, diastólica $\geq 85 \mathrm{mmHg}$, uso de medicação anti-hipertensiva; ${ }^{* * * *}$ Glicemia de jejum elevada: $\geq 100 \mathrm{mg} / \mathrm{dL}$ ou diagnóstico médico de diabetes tipo $2 ;{ }^{* * * * *}$ Hipertrigliceridemia: $>150 \mathrm{mg} / \mathrm{dL}$ em jejum; ${ }^{* * * * *} \mathrm{HDL}-\mathrm{C}$ baixo: $<40 \mathrm{mg} / \mathrm{dL}$ em homens e $<50 \mathrm{mg} / \mathrm{dL}$ em mulheres, em jejum.

Tabela 4. Odds ratio (IC 95\%) de apresentar hipertensão arterial sistêmica, diabetes mellitus e síndrome metabólica nos diferentes níveis de estado nutricional. Marques de Souza (RS), 2007.

\begin{tabular}{lccc}
\hline Característica & Magreza $n=34$ & Normal $n=73$ & Excesso de peso $n=92$ \\
\hline HAS & $0,42(0,17$ a 1,06) & 1 & $3,3(1,70$ a 6,4$)$ \\
Diabetes mellitus & $0,37(0,08$ a 1,78) & 1 & $3,3(1,48$ a 7,1$)$ \\
Síndrome metabólica & - & 1 & $6,9(3,2$ a 14,6) \\
\hline
\end{tabular}

1 Odds ratio (IC de 95\%) calculado por modelo de regressão logística ajustado por idade e sexo, utilizando o grupo "Normal" como referência. IMC: índice de massa corporal; HDL: lipoproteína de alta densidade; LDL: lipoproteína de baixa densidade: HAS: hipertensão arterial sistêmica; PA: pressão arterial. 
prevalências de HAS, DM e SM foram progressivamente maiores naqueles com excesso de peso quando comparados aos com estado nutricional magreza e normal, independentemente do gênero e da idade.

Dentre os parâmetros antropométricos utilizados para avaliação do estado nutricional, o mais utilizado na prática clínica atualmente é o IMC, por ser prático, de manejo simples e capaz de identificar facilmente a obesidade generalizada. Segundo Lipschitz ${ }^{3}$, considera-se excesso de peso idosos com níveis de IMC acima de $27 \mathrm{~kg} / \mathrm{m}^{2}$.

Em adultos, valores de IMC acima de $25 \mathrm{~kg} / \mathrm{m}^{2}$ estão associados, de forma significativa, com maior risco de DCV, de forma independente do Escore de Framingham, e com aumento de morbidade, principalmente relacionada à DM e DCV. Níveis de IMC acima de $30 \mathrm{~kg} / \mathrm{m}^{2}$ levam a um aumento de mortalidade e morbidade associadas à DM, DCV e acidente vascular cerebral ${ }^{9}$. O IMC não perde seu valor na avaliação de risco cardiovascular também quando associado à medida da obesidade abdominal ${ }^{10}$. Em idosos pouco se tem estudado em relação aos pontos de corte do IMC para determinação de excesso de peso e sua associação com riscos à saúde. Assim sendo, valores inadequados poderiam estar sendo utilizados para pacientes geriátricos.

Neste estudo, a prevalência de excesso de peso nos idosos foi de $46 \%$ ( $42 \%$ nos homens e $50 \%$ nas mulheres), inferior à prevalência encontrada entre idosos chineses, de $56 \%$ (54\% nos homens e $58 \%$ nas mulheres) ${ }^{11}$. As diferentes categorias de estado nutricional avaliadas pelo IMC, conforme esperado, apresentaram associação com a prevalência dos componentes para SM de obesidade central, pressão arterial elevada, glicemia elevada e hipertrigliceridemia em ambos os gêneros, com os idosos com excesso de peso apresentando maior prevalência dessas doenças quando comparados com os idosos de IMC normal e magreza. Já em relação ao HDL-C baixo, foi encontrada associação com as categorias de IMC apenas entre os homens. Talvez tenha faltado poder estatístico, com o pequeno número de mulheres em cada faixa de estado nutricional, não possibilitando encontrar associação com níveis de HDL-C baixo. A prevalência de hipertensão arterial, diabetes mellitus e de síndrome metabólica também foram significativamente superior entre os idosos com excesso de peso.

Encontramos diferença significativa na prevalência de SM entre os gêneros: em $26 \%$ dos homens e em $44 \%$ das mulheres. Um estudo australiano, utilizando IMC $>29,3 \mathrm{~kg} / \mathrm{m}^{2}$ no lugar da circunferência abdominal elevada para diagnosticar SM, encontrou $31 \%$ de homens e $34 \%$ de mulheres com presença da síndrome ${ }^{12}$, mas a diferença no critério diagnóstico poderia justificar a diferença de nossos resultados. Em outro estudo, em uma população de idosos chineses, utilizando os critérios do IDF, a prevalência de SM em homens foi de $35 \%$ e em mulheres de $54 \%$, resultados semelhantes aos nossos ${ }^{11}$.

A razão de chances de os idosos com excesso de peso apresentarem HAS, DM ou SM foi várias vezes maior do que aqueles com estado nutricional normal, independentemente do gênero e da idade. Estudos prévios salientaram a associação da SM com doença cardiovascular aterosclerótica, apresentando os portadores dessa síndrome maior incidência de eventos coronarianos ${ }^{13,14}$. No presente estudo, os idosos com excesso de peso tiveram razão de chances de 6,9 (IC95\% 3,2 a 14,6) de apresentar SM em relação àqueles com estado nutricional normal. Entre os idosos magros não foi encontrado nenhum caso de SM.

Existe menor correlação entre IMC e mortalidade em idosos em comparação à encontrada em pessoas jovens ${ }^{6}$, possivelmente devido às alterações corporais do envelhecimento ou pelo fato de idosos já serem sobreviventes, tendo aqueles mais suscetíveis a esse fator de risco morrido anteriormente. Também o peso de outros tradicionais fatores de risco para DCV, como o colesterol, muda nessa faixa etária ${ }^{15-16}$. Níveis de IMC entre 25 e $27 \mathrm{~kg} / \mathrm{m}^{2}$ não são considerados fator de risco para mortalidade cardiovascular e morte por todas as causas na população idosa de ambos 
os gêneros. Em uma revisão sistematizada, a maioria dos estudos avaliados mostra associação negativa ou ausente entre IMC e todas as causas de morte em idosos, ao contrário do mostrado para jovens e adultos jovens. Nessa metanálise, poucos estudos evidenciaram valores de IMC iguais ou acima de $27 \mathrm{~kg} / \mathrm{m}^{2}$ como fator prognóstico para mortalidade cardiovascular e por todas as causas em pessoas de 65 a 74 anos, com apenas um demonstrando que níveis de IMC iguais ou acima de $28 \mathrm{~kg} / \mathrm{m}^{2}$ estiveram associados a maior risco de morte em pacientes com mais de 75 $\operatorname{anos}^{17}$. Em estudo recente em população chinesa, o IMC foi fortemente associado ao aumento da prevalência de doenças cardiovasculares, independentemente da presença de SM.

A escolaridade e a renda não apresentaram associação significativa com o estado nutricional dos idosos estudados. No que se refere à escolaridade, observamos que a maioria dos idosos deste estudo tem apenas o ensino fundamental incompleto, semelhante ao que Pereira ${ }^{18}$ também encontrou no Rio Grande do Sul, com 71,0\% de idosos com menos de quatro anos de estudo. Em relação à renda, foi encontrada a maioria dos idosos vivendo com um a três salários-mínimos per capita; e 37,0\% dos homens e 44,0\% das mulheres com menos de um salário-mínimo. Esses dados corroboram os da população brasileira de 2003, que revelam 43,8\% dos idosos com rendimento inferior a um salário-mínimo per capita ${ }^{19}$.

Já quanto ao local de moradia, os percentuais de homens e mulheres idosos com excesso de peso foram maiores na área urbana do que na rural, mas sem diferença significativa. A atividade física exercida por esses idosos pode ter influenciado a diferença de estado nutricional encontrada entre eles. A maioria dos idosos da área rural ainda se dedica ao trabalho agrícola, enquanto os da área urbana são mais sedentários. Em estudo de Tavares e Anjos, com base nos dados da Pesquisa Nacional sobre Saúde e Nutrição realizada em 1989, foi observado que o sobrepeso e a obesidade também eram mais prevalentes em homens e mulheres nas áreas urbanas de todas as regiões do Brasili20.

\section{O N C L US Ã O}

Segundo este estudo, em idosos, o diagnóstico de excesso de peso está associado a importante risco cardiovascular, pois encontramos forte associação entre o estado nutricional determinado pelo IMC e fatores de risco já estabelecidos. Embora o aumento de mortalidade cardiovascular só possa ser demonstrado em estudos longitudinais, nossos achados em estudo transversal sugerem que, em idosos, o diagnóstico de excesso de peso com a utilização do IMC pode ajudar a definir um subgrupo sob maior risco, de maneira simples e eficaz, o que poderá ajudar na formulação de políticas públicas que desenvolvam a prevenção de DCV nessa população com menor custo operacional e uma visão interdisciplinar dos profissionais envolvidos. Pode-se incluir, portanto, como rotina do processo de triagem de pacientes atendidos no serviço público, a determinação do IMC a fim de detectar precocemente os riscos aumentados de DCV.

\section{COLABORADORES}

F. SCHERER responsável pela concepção, desenho e pela aquisição dos dados do estudo e pela redação do artigo. J.L.C. VIEIRA responsável pelo desenho do artigo, pela análise e pela interpretação dos dados e pela redação do artigo.

\section{REFER Ê N CIAS}

1. Yusuf S, Hawken S, Ounpuu S, Dans T, Avezum A, Lanas $F$, et al. Effect of otentially modifiable risk factors associated with myocardial infarction in 52 countries (the INTERHEART study): case-control study. Lancet. 2004; 364(9438):937-52. doi: 10.10 16/S0140-6736(04)17018-9.

2. Hayman LL, Meininger JC, Daniels SR, McCrindle BW, Helden L, Ross J, et al. Primary prevention of cardiovascular disease in nursing practice: focus on children and youth. Circulation. 2007; 116(3): 344-57.

3. Alberti KG, Zimmet P, Shaw J. The metabolic syndrome: a new worldwide definition. Lancet. 2005; 366(9491):1059-62. doi:10.1016/S0140-67 36(05)67402-8. 
4. I Diretriz da Síndrome Metabólica. Rev Soc Bras Hipert. 2004; 7(4):123-60.

5. Ramos R, Rosa TE, Oliveira ZM, Medina MC, Santos FR. Perfil do idoso em área metropolitana na região sudeste do Brasil: resultados de inquérito domiciliar. Rev Saúde Pública. 1993; 27(2):87-94.

6. McTigue KM, Hess R, Ziouras J. Obesity in older adults: a systematic review of the evidence for diagnosis and treatment. Obesity (Silver Spring). 2006; 14(9):1485-97.

7. Cabrera MAS, Filho WJ. Obesidade em idosos: prevalência, distribuição e associação com hábitos e comorbidades. Arq Bras Endocrinol Metab. 2001; 45(5):494-501.

8. Lipschitz DA. Screening for nutritional status in the elderly. Prim Care. 1994; 21(1):55-67.

9. Mora S, Yanek LR, Moy TF, Fallin MD, Becker LC, Becker DM. Interaction of body mass index and framingham risk score in predicting incident coronary disease in families. Circulation. 2005; 111(15):1871-6. doi: 10.1161/01.CIR.0000161 956.75255.7B.

10. Janssen I, Heymsfield SB, Allison DB, Kotler DP, Ross R. Body mass index and waist circumference independently contribute to the prediction of nonabdominal, abdominal subcutaneous, and visceral fat. Am J Clin Nutr. 2002; 75(4):683-8.

11. He $Y$, Jiang B, Wang J, Feng $K$, Chang Q, Zhu S, et al. BMI versus the metabolic syndrome in relation to cardiovascular risk in elderly Chinese individuals. Diabetes Care. 2007; 30(8):2128-34. doi: 10.2337/ dc06-2402.

12. Simons LA, Simons J, Friedlander Y, McCallum J. Does a diagnosis of the metabolic syndrome provide additional prediction of cardiovascular disease and total mortality in the elderly? The Dubbo Study. Med J Aust. 2007;186(8):400-3. doi: 10.1007/s00125-009-1271-5.
13. Ford ES. The metabolic syndrome and mortality from cardiovascular disease and all-causes: findings from the National Health and Nutrition Examination Survey II Mortality Study. Atherosclerosis. 2004; 173(2):309-14.

14. Isomaa $B$, Almgren $P$, Tuomi $T$, Forsen $B$, Lahti $K$, Nissen $\mathrm{M}$, et al. Cardiovascular morbidity and mortality associated with the metabolic syndrome. Diabetes Care. 2001; 24(4):683-9. doi: 10.1007/s1 1126-008-9088-9.

15. Fried LP, Kronmal RA, Newman AB, Bild DE, Mittelmark MB, Polak JF, et al. Risk factors for 5-year mortality in older adults: the cardiovascular health study. JAMA. 1998; 279(8):585-92.

16. Psaty BM, Furberg CD, Kuller LH, Bild DE, Rautaharju PM, Polak JF, et al. Traditional risk factors and subclinical disease measures as predictors of first myocardial infarction in older adults: the cardiovascular health study. Arch Intern Med. 1999; 159(12):1339-47.

17. Heiat A, Vaccarino V, Krumholz HM. An evidencebased assessment of federal guidelines for overweight and obesity as they apply to elderly persons. Arch Intern Med. 2001; 161(9):1194-203.

18. Pereira RJ. Contribuição dos domínios físico, social, psicológico e ambiental para a qualidade de vida global de idosos. Rev Psiquiatr. 2006; 28(1):27-38. doi: 10.1590/S1517-86922009000300002.

19. Instituto Brasileiro de Geografia e Estatística. Síntese de indicadores sociais 2004. Rio de Janeiro: IBGE; 2005.

20. Tavares EL, Anjos LA. Perfil antropométrico da população idosa brasileira. Resultados da Pesquisa Nacional sobre Saúde e Nutrição. Cad Saúde Pública. 1999; 15(4):759-68.

Recebido em: 28/7/2008

Versão final reapresentada em: 14/7/2009 Aprovado em: 18/3/2010 